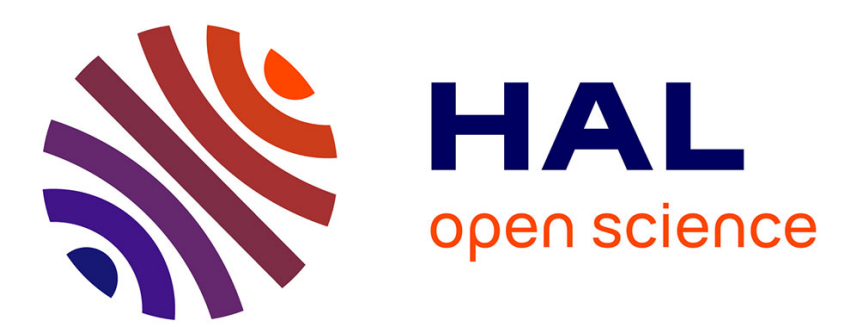

\title{
Noise-robust Stress Intensity Factor Determination from Kinematic Field Measurements
}

\author{
Julien Réthoré, Stéphane Roux, François Hild
}

\section{To cite this version:}

Julien Réthoré, Stéphane Roux, François Hild. Noise-robust Stress Intensity Factor Determination from Kinematic Field Measurements. Engineering Fracture Mechanics, 2008, 75 (13), pp.3763-3781. 10.1016/j.engfracmech.2007.04.018 . hal-00200631

\section{HAL Id: hal-00200631 \\ https://hal.science/hal-00200631}

Submitted on 11 Jan 2008

HAL is a multi-disciplinary open access archive for the deposit and dissemination of scientific research documents, whether they are published or not. The documents may come from teaching and research institutions in France or abroad, or from public or private research centers.
L'archive ouverte pluridisciplinaire HAL, est destinée au dépôt et à la diffusion de documents scientifiques de niveau recherche, publiés ou non, émanant des établissements d'enseignement et de recherche français ou étrangers, des laboratoires publics ou privés. 


\title{
Noise-robust Stress Intensity Factor Determination from Kinematic Field Measurements
}

\author{
Julien Réthoré ${ }^{\mathrm{a}}$ Stéphane Roux ${ }^{\mathrm{b}, *}$ François Hild $^{\mathrm{c}}$ \\ ${ }^{a}$ Delft University of Technology \\ Faculty of Aerospace Engineering \\ P.O. Box 5058, 2600 GB Delft, The Netherlands \\ ${ }^{\mathrm{b}}$ Surface du Verre et Interfaces \\ UMR CNRS / Saint-Gobain \\ 39 Quai Lucien Lefranc, 93303 Aubervilliers Cedex, France \\ ${ }^{\mathrm{c}}$ Laboratoire de Mécanique et Technologie (LMT-Cachan) \\ Ecole Normale Supérieure de Cachan / UMR CNRS / Université Paris 6 \\ 61 Avenue du Président Wilson, 94235 Cachan Cedex, France
}

\begin{abstract}
Stress Intensity Factors are often estimated numerically from a given displacement field through an interaction integral formalism. The latter method makes use of a weight, the virtual crack extension field, which is under-constrained by first principles. Requiring a least noise sensitivity allows one to compute the optimal virtual crack extension. Mode $I$ and mode $I I$ specialized fields are obtained and particularized for a given displacement functional basis. The method is applied to an experimental case study of a crack in a silicon carbide sample, whose displacement field is obtained by a digital image correlation technique. The optimization leads
\end{abstract}


to a very significant uncertainty reduction up to a factor 100 of the non-optimized formulation. The proposed scheme reveals additional performances with respect to the integral domain choice and assumed crack tip geometry, which are shown to have a reduced influence.

Key words: digital image correlation, interaction integral, uncertainty, stress intensity factor

\section{Introduction}

Full field measurement techniques now provide reliable experimental data for displacement fields from which stress intensity factors may be estimated (McNeill et al., 1987; Anbanto-Bueno and Lambros, 2002; Roux and Hild, 2006; Yoneyama et al., 2006). In these references, least squares minimization is used to identify stress intensity factors from theoretical displacement fields around a crack tip. An alternative technique to determine stress intensity factors is to evaluate directly the $J$-integral along a given contour (Huntley and Field, 1989). However, only few measurement data are used and strains and stresses are to be computed. To avoid this drawback, interaction integrals are used for extracting stress intensity factors from a discrete displacement field. This technique was first developed in the framework of finite element calculations (Suo and Combescure, 1992; Goz et al., 1998; Attigui and Petit, 1997; Rajaram et al., 2000; Moës et al., 2002; Réthoré et al., 2005a) and more recently for experimental identification using digital image correlation

\footnotetext{
* Surface du Verre et Interfaces, UMR CNRS / Saint-Gobain, 39 Quai Lucien Lefranc, 93303 Aubervilliers Cedex, France

Email address: stephane.roux@saint-gobain.com (Stéphane Roux).
} 
measurements (Réthoré et al., 2005b). The interests of the interaction integral when used in combination with kinematic field measurements are the theoretical domain invariance and the ability to extract the singularity without an a priori determination of the $K$-dominant zone. Another advantage is its robustness with respect to the presence of a process zone in the vicinity of the crack tip, even if the latter violates the assumptions of Linear Elastic Fracture Mechanics.

However, a difficulty is that the interaction integral involves the gradient of the displacement field that may display large uncertainty levels. Therefore, a high noise sensitivity of the stress intensity factors estimation by this technique is expected (and observed). The aim of this paper is to exploit the not so constrained virtual crack extension (VCE) field of the interaction integral to improve its robustness to measurement uncertainties. A general formulation is proposed to optimize this field in order to minimize the effect of a measurement uncertainty. Moreover, the proposed procedure is tailored to the discretization at hand, and thus to the displacement functional basis.

First, the interaction integral is written in a discrete form that matches the discretized displacement field, irrespective of its origin. Yet, at this stage, the virtual crack extension field is kept unspecified. Then the influence of a measurement uncertainty is studied and quantified. The optimized virtual crack extension field is constructed and the stress intensity factors are computed. The performances of the proposed approach will be evaluated on a real experimental case. The application discussed herein is based upon a Silicon Carbide sandwiched beam crack test (Nose and Fujii, 1988; Marshall et al., 1991; Pancheri et al., 1998; Forquin et al., 2004). This type of experimental set-up allows one to initiate a crack without complete failure of the central 
beam. However, the arrest conditions are strongly dependent upon the friction between the beams, their flexural stiffness, the notch geometry. Therefore, a stress intensity factor identification using finite element simulations of the experiments was shown to be unreliable (Forquin et al., 2004). Consequently, a direct identification such as the one proposed herein, is the only means to analyze this type of experiment.

\section{Interaction integral}

Let us start with the interaction integral $I^{\text {int }}$ defined in (Réthoré et al., 2005b). The co-ordinate system is centered at the crack tip and the unit vector $\mathbf{x}$ is given by the crack direction (the crack lies along the negative $x$-axis, see Fig. 1). A displacement field $\mathbf{u}$ is assumed to be known and the corresponding stress field $\sigma$ derives from a linear elastic constitute law with known elastic constants. Auxiliary displacement and stress fields, $\mathbf{u}^{\text {aux }}$ and $\sigma^{\text {aux }}$, (related through the same constitutive equation) and the so-called virtual crack extension field $\mathbf{q}$ are introduced. The interaction integral $I^{\text {int }}$ reads

$$
I^{i n t}=-\int_{\Omega}\left[\sigma_{m l}^{a u x} u_{m, l} \delta_{k j}-\left(\sigma_{i j}^{a u x} u_{i, k}+\sigma_{i j} u_{i, k}^{a u x}\right)\right] q_{k, j} \mathrm{~d} \Omega
$$

where $\delta_{k j}$ is the Kronecker operator. In this expression, the VCE field q simply appears as a (vector) weight function. It is assigned a value $\mathbf{x}$ at the tip and $\mathbf{0}$ on the boundary of the integration domain $\partial \Omega$. It is also orthogonal to the 
crack faces

$$
\begin{aligned}
\mathbf{q} \cdot \mathbf{n}_{\Gamma}=0 & \text { on } \Gamma \\
\mathbf{q}=\mathbf{x} & \text { at the tip } \\
\mathbf{q}=\mathbf{0} & \text { on } \partial \Omega
\end{aligned}
$$

where $\Gamma$ is the geometrical support of the crack and $\mathbf{n}_{\Gamma}$ is the normal to $\Gamma$.

If the crack faces are traction-free for both actual and auxiliary fields, then $I^{\text {int }}$ is shown to be domain-independent (Suo and Combescure, 1992; Goz et al., 1998). Furthermore, if both fields satisfy the balance of momentum equation under a plane stress assumption then going to the limit of an integration domain reduced to the crack tip, $I^{\text {int }}$ becomes

$$
I^{i n t}=\frac{2}{E}\left(K_{I} K_{I}^{a u x}+K_{I I} K_{I I}^{a u x}\right)
$$

where $K_{i}$, respectively $K_{i}^{\text {aux }}$, are the stress intensity factors of the actual, respectively auxiliary, fields. Consequently, one computes $K_{I}$ and $K_{I I}$ choosing the auxiliary field to be the Westergaard solution for a cracked body in mode $I$ and $I I$ with a unit stress intensity factor.

The actual displacement field is now assumed to be approximated on a functional basis $\boldsymbol{\Psi}_{\mathbf{u}}$ that will be discussed more extensively in Section 4.1. The contributions $U_{i}$ of each function $\Psi_{\mathbf{u} i}$ are collected in a vector $\mathbf{U}$. This approximation is the result of a kinematic field measurement and the actual displacement field then reads

$$
\mathbf{u}=\Psi_{\mathbf{u}} \mathbf{U}
$$

An interpolation basis is also chosen for the weight function and using the same notation, one obtains

$$
\mathrm{q}=\Psi_{\mathrm{q}} \mathrm{Q}
$$


Inserting Eqs. (4) and (5) into Eq. (1), a generic stress intensity factor $K$ (for mode $I$ or mode $I I$ ) is given by Eq. (3)

$$
K=\mathbf{Q}^{\mathrm{T}} \mathbf{M U}
$$

In this equation, $K$ and $\mathbf{M}$ are specialized for mode $I$ or mode $I I$ by selecting the appropriate auxiliary field

$$
\mathbf{M}=-\frac{E}{2} \int_{\Omega} \nabla \boldsymbol{\Psi}_{\mathbf{q}}^{\mathrm{T}}\left[\boldsymbol{\sigma}^{a u x}: \nabla \boldsymbol{\Psi}_{\mathbf{u}} \mathbf{I}-\left(\boldsymbol{\sigma}^{a u x} \nabla \boldsymbol{\Psi}_{\mathbf{u}}+\left(\mathbf{D} \nabla^{s} \boldsymbol{\Psi}_{\mathbf{u}}\right) \nabla \mathbf{u}^{a u x}\right)\right] \mathrm{d} \Omega
$$

where $\mathbf{D}$ is the fourth-order stiffness tensor, $\mathbf{I}$ the second order identity tensor and $\nabla^{s}$ the symmetric strain operator.

\section{Noise sensitivity}

Let us assume that the actual approximated displacement field is corrupted by a random (vector) noise $\boldsymbol{\eta}$ of zero mean

$$
\langle\boldsymbol{\eta}\rangle=\mathbf{0}
$$

This noise is also characterized by a correlation kernel $\mathbf{C}$, here normalized so that its magnitude is denoted through the (scalar) variance of one component. Hence, the covariance tensor of the noise vector field reads

$$
\langle\boldsymbol{\eta} \otimes \boldsymbol{\eta}\rangle=\mathbf{C} \eta^{2}
$$

For an uncorrelated white noise $C_{i j}=\delta_{i j}$ and the variance of each component of the noise is $\eta^{2}$.

This measurement uncertainty affects the estimation of the stress intensity factors. Using the linearity of Eq. (6), the perturbation $\delta K$ induced by the 
noise $\boldsymbol{\eta}$ is of zero mean

$$
\langle\delta K\rangle=\mathbf{Q}^{\mathrm{T}} \mathbf{M}\langle\boldsymbol{\eta}\rangle=0
$$

and its variance reads

$$
\left\langle\delta K^{2}\right\rangle=\mathbf{Q}^{\mathrm{T}} \mathbf{M C M}^{\mathrm{T}} \mathbf{Q} \eta^{2}
$$

From this equation, the variance $\left\langle\delta K^{2}\right\rangle$ depends upon the nodal values of the VCE field, both displacement and weight function functional basis, the auxiliary field and the noise kernel. However, it is independent of the measured displacement field. Equation (10) shows that if the measured displacements are not biased, then the stress intensity factor estimate is unbiased as well. This is not the case when using directly the $J$-integral, which is a quadratic form of the actual displacement field, leading to systematic errors in the determination of $K_{I}$. Last, an interesting feature of Eq. (11) is that the $K$ variance is expressed as a quadratic form of $\mathbf{Q}$. Hence, it can easily be exploited for a minimization.

\section{Optimal virtual crack extension field}

\subsection{Numerical elaboration}

Linear type functions, as plotted in Fig. 2, are usually used as weight function in the interaction integral for finite element applications (Suo and Combescure, 1992; Goz et al., 1998; Réthoré et al., 2005a) or in an experimental

framework (Réthoré et al., 2005b). The fact that $\mathbf{q}$ is only constrained by Eq. (2) is exploited to reduce the noise sensitivity of the stress intensity factor identification. For a given basis of interpolation functions, $\boldsymbol{\Psi}_{\mathbf{u}}$, an optimal weight function is computed on the functional basis $\boldsymbol{\Psi}_{\mathrm{q}}$ using the minimization 
of Eq. (11) for mode $I$ and mode $I I$. The essential boundary conditions (2) for the weight function are prescribed using Lagrange multipliers, denoted collectively as a vector $\boldsymbol{\lambda}$

$$
\left[\begin{array}{cc}
\mathrm{MCM}^{\mathrm{T}} & \mathbf{L}^{\mathrm{T}} \\
\mathbf{L} & 0
\end{array}\right]\left(\begin{array}{l}
\mathrm{Q} \\
\boldsymbol{\lambda}
\end{array}\right)=\left(\begin{array}{c}
0 \\
\mathrm{Q}_{p}
\end{array}\right)
$$

The solution to this system is an optimal weight function since it minimizes the sensitivity of the estimation of $K$ with respect to measurement uncertainties. The matrix $\mathbf{L}$ is the linking matrix and $\mathbf{Q}_{p}$ collects the prescribed value of q. Above, we proposed a general set of equations that may suggest that a unique virtual crack extension field holds for any mode. Although this could be envisioned, it is here proposed to design specific optimal VCE focused either on mode $I$ or mode $I I$.

Preliminary tests led to the conclusion that the interpolation basis for the displacement field had to be consistent with the crack support. Since the interaction integral involves a strain interaction energy between the actual and the auxiliary field, continuous functions across the crack faces introduce spurious components. Consequently, the interpolation basis is built in the spirit of the extended finite element method. It includes standard finite element shape functions, discontinuous functions and singular functions in the vicinity of the crack tip. Following Moës et al. (1999), the interpolation of the displacement field is enriched for each component $u_{k}$

$$
u_{k}=\sum_{i \in \mathcal{N}} N_{i} u_{i}+\sum_{i \in \mathcal{N}_{\text {cut }}} N_{i} \mathcal{H}_{\Gamma} a_{i}+\sum_{i \in \mathcal{N}_{\text {tip }}} \sum_{j \in[1 ; 4]} N_{i} B_{j} b_{i j}
$$

where $N_{i}$ are standard finite element shape functions supported by the set of nodes $\mathcal{N}$ included in the discretized domain $\Omega$. Nodes in $\mathcal{N}_{\text {cut }}$ have their 
support completely cut by the crack (Fig. 3). They hold additional degrees of freedom $a_{i}$ corresponding to the discontinuous function $\mathcal{H}_{\Gamma}$ defined by

$$
\mathcal{H}_{\Gamma}=\left\{\begin{aligned}
1 & \text { above the crack } \\
-1 & \text { below the crack }
\end{aligned}\right.
$$

Nodes whose support contains the crack tip are included in $\mathcal{N}_{\text {tip }}$. Four singular functions $B_{j}$ and their corresponding degrees of freedom $b_{i j}$ are supported by this set of node. The singular functions are given by

$$
\left\{B_{j}(r, \theta)\right\}_{j \in[1 ; 4]}=\left\{\sqrt{r} \cos \frac{\theta}{2}, \sqrt{r} \cos \frac{\theta}{2} \sin \theta, \sqrt{r} \sin \frac{\theta}{2}, \sqrt{r} \sin \frac{\theta}{2} \sin \theta\right\}
$$

where $r, \theta$ are the polar crack tip co-ordinates. Then, the displacement functional basis $\boldsymbol{\Psi}_{\mathbf{u}}$ reads

$$
\Psi_{\mathbf{u}}=\left\{N_{i}\right\}_{i \in \mathcal{N}} \cup\left\{N_{i} \mathcal{H}_{\Gamma}\right\}_{i \in \mathcal{N}_{\text {cut }}} \cup\left\{N_{i} B_{j}\right\}_{i \in \mathcal{N}_{\text {tip }}, j \in[1 ; 4]}
$$

In the experimental test, the displacement field was determined using a digital image correlation technique and bi-linear quadratic finite element shape functions $N_{i}$ (Besnard et al., 2006) without any a priori assumption on the crack location. Thus a re-projection step is necessary for this consistency requirement. The details of this correction are presented in Section 5.1. Concerning the weight function, $\mathbf{q}$ is discretized on a standard finite element basis

$$
\Psi_{\mathbf{q}}=\left\{N_{i}\right\}_{i \in \mathcal{N}}
$$

\subsection{Results}

For the functional bases (16) and (17), the system (12) is solved to obtain the optimal weight function. In a first step, it is assumed that $\mathbf{q}$ complies with the 
standard choice of a vector field parallel to $\mathbf{x}$

$$
\mathbf{q}=q \mathbf{x}
$$

so that $q$ is reduced here to a scalar amplitude. The noise is considered to be uncorrelated. The optimal weight functions obtained for mode $I$ and mode II are plotted in Fig. 4. The difference between the optimal weight functions and a cone is significant (Fig. 2). Let us also note that each weight function is slightly, but definitely, different.

The ratio between the variance $\left\langle\delta K^{2}\right\rangle$ (computed using Eq. (11)) for the optimal function and the conical function are plotted in Fig. 5 for both modes. Let us note the very large reduction in variance for the stress intensity factor, which reaches values as low as 0.017 and still decreases with the size of the integration domain.

The decrease of the variance with the domain size is further characterized. First let us mention that a theoretical continuous formulation of the above problem can be written, leading to a fourth order (inhomogeneous) partial differential equation. This is of little practical interest since the above procedure naturally produces a discretization of this equation that is ideally adapted to the problem at hand. However, it indicates that there exists a well defined limit when the domain size is large compared to the mesh size. Using this property, we may introduce the continuum limit $\operatorname{VCE} \rho(x)$ such that for a large domain size, $a, q(x)=\rho(x / a)$. Figure 6 shows the optimal VCE functions in the plane $x=0$ for a domain size $a=400 \mu \mathrm{m}, a=600 \mu \mathrm{m}$ and $a=750 \mu \mathrm{m}$. These results show that the continuum limit VCE is closely obtained for domain sizes corresponding to about 20,30 and 37 times the characteristic element size. 
It is possible to get some more insight into the scaling properties of the variance of $K$ with respect to the domain size, $a$, from dimensional analysis. By exploiting the algebraic singularity of the reference stress and strain field, and integrating over the angular variable, the expression of the variance is proportional to the following integral

$$
\left\langle\delta K^{2}\right\rangle \propto(1 / a)^{2} \int_{0}^{a} r^{-2} \rho^{\prime}(r / a)^{2} \mathrm{~d} r
$$

For a cone, the contribution of the noise coming from the origin is singular, and hence, the above integral is divergent. Its discretized version is therefore dominated by the first elements, and hence $\left\langle\delta K^{2}\right\rangle \propto(1 / a)^{2}$. The minimization of the above quantity with respect to $\rho$ thus implies that $\rho^{\prime}(r / a)$ vanishes at the origin, i.e. the optimal VCE function should be parabolic close to the crack tip, as shown in Fig. 6. The integrand being well behaved, the scaling reads

$$
\left\langle\delta K^{2}\right\rangle \propto(1 / a)^{3} \int_{0}^{1} u^{-2} \rho^{\prime}(u)^{2} \mathrm{~d} u \propto a^{-3}
$$

This property is shared by all VCE fields which are parabolic at the crack tip

Both results are confirmed by Fig. 7 where the variances are plotted for conical and optimized VCEs as functions of the domain size. Moreover, from this argument, one predicts that the ratio of the noise variances of the optimum to the cone VCE weight should decrease with the domain size as $1 / a$, as observed in Fig. 5.

\subsection{Test with alternate functions}

Examining the shape of the optimal weight functions and considering the variation of the variance with the domain size, one studies different weight 
functions. First, a truncated cone where $\mathbf{q}$ is constant and equal to $\mathbf{x}$ in a region whose size is, for example, half the size of the integration domain. As observed in Fig. 8, the plateau improves the noise robustness of the cone weight function and the reduction obtained with the optimal weight function is now below 0.4 .

Second, a particular function was used in planes orthogonal to a 3-dimensional crack fronts (Rajaram et al., 2000), denoted as $q_{R S P}$ after the initials of the authors' names, and referred to as RSP function in the sequel. It is used to analyze in-plane displacements and the virtual field reads

$$
q_{R S P}=e^{-\left(\frac{r}{r-r_{\max }}\right)^{2}}
$$

where $\sqrt{2} r_{\max }$ is the radius of influence of this function. It is worth noting that this function has a shape similar to the optimal VCE proposed herein, namely, it has zero derivatives at the crack tip and also on $\partial \Omega$. Its shape is similar to the optimized VCE in the plane $x=0$. However, examining Fig. 4, one observes that the shape of optimized VCE does not hold any symmetry except with respect to the crack plane, in contrast to the RSP weight. Furthermore, the optimized VCE accounts for the specificity of modes $I$ and $I I$ leading to two distinct functions. Because the RSP function is not optimal with respect to noise, its noise sensitivity is greater than that of the optimal VCE, as shown in Fig. 7, although both display the same scaling with $a$ since their derivative vanish at the crack tip. The ratio of their variance is about $1 / 2$. Furthermore, for small sized domains the performances of the RSP function are close to those of the conical VCE. This may be due to the interpolation of the RSP function with bilinear finite element shape functions that is inherent in the proposed determination of noise sensitivity. This effect is supposed to be negligible for large domain sizes and hence it does not affect 
the results obtained with the RSP function for large sizes of the integration domain (i.e.greater than $500 \mu \mathrm{m})$.

\subsection{Influence of noise correlation}

The performance of the digital image correlation technique used herein has been evaluated by Besnard et al. (2006). The displacement uncertainty and the noise resulting from the correlation technique are described in that paper. The noise correlation matrix was computed to be

$$
\begin{array}{ll}
C_{i j}=1 & \text { if } i=j \\
C_{i j}=-\frac{1}{4} & \text { if } i \text { and } j \text { are first neighbor nodes } \\
C_{i j}=\frac{1}{16} & \text { if } i \text { and } j \text { are second neighbor (diagonally opposed) nodes }
\end{array}
$$

For this correlated noise, the noise sensitivity is plotted in Fig. 9. The reduction assessed by the optimization of the weight function is more significant. Since the optimization depends upon the displacement functional basis $\boldsymbol{\Psi}_{\mathbf{u}}$ and also upon the correlation matrix $\mathbf{C}$, the optimal weight function takes care of the correlated nature of noise. Hence, the improvement achieved by the optimal weight function is even higher (up to a factor 100 for a box size of $600 \mu \mathrm{m}$ ).

\section{Stress intensity factors estimation}

The test case discussed hereafter is based upon a Silicon Carbide sandwiched beam test (Fig. 10-a). The SiC beam has a length of $25 \mathrm{~mm}$, a height of $5 \mathrm{~mm}$ and a width of $3 \mathrm{~mm}$. The outer span is equal to $20 \mathrm{~mm}$. Additional details can be found in (Forquin et al., 2004). The kinematic measurement (see Fig. 11) 
are carried out by using a Q4P1 correlation algorithm (Besnard et al., 2006) on the pictures of Fig. 10. The mesh consists of 1190 four-node elements (i.e. Q4) of length 16 pixels over an area of interest of $944 \mu \mathrm{m} \times 971 \mu \mathrm{m}$ with a conversion factor $p=1.85 \mu \mathrm{m} /$ pixel. The displacement field is obtained on a linear (P1) finite element shape function basis. The crack tip position is supposed to be determined by a previous analysis (Roux and Hild, 2006). The linear elastic behavior of the Silicon Carbide is characterized by a Young's modulus $E=410 \mathrm{GPa}$ and Poisson's ratio $\nu=0.15$.

\subsection{Kinematic projection}

In a similar way as for the noise sensitivity of the estimation of the stress intensity factors, the stress intensity factors themselves are identified by using Eq. (6) after the weight function is determined. For this purpose, the displacement field initially obtained on a standard finite element shape functions basis has to be projected onto the functional basis $\boldsymbol{\Psi}_{\mathbf{u}}$ used in the determination of the weight function. The projection is only needed for the nodes whose support is cut by the crack because the enhanced functions are active only in this region (see Eq. (13)). The preferred solution consists in a mechanical projection based on the weak form of the following constraint

$$
\begin{aligned}
\sigma_{i j, j}=0 & \text { over } \Omega_{\Gamma} \\
\sigma\left(\mathbf{n}_{\Gamma}\right)=0 & \text { on } \Gamma \\
\mathbf{u}=\mathbf{u}_{p} & \text { on } \partial \Omega_{\Gamma}
\end{aligned}
$$

where $\Omega_{\Gamma}$ is the region containing the elements cut by the crack plus one more element layer and $\mathbf{u}_{p}$ the displacement obtained by digital image correlation 
on the boundary $\partial \Omega_{\Gamma}$ of $\Omega_{\Gamma}$ (see Fig. 3). Then, a standard static analysis with the extended basis of shape functions $\boldsymbol{\Psi}_{\mathbf{u}}$ is performed over $\Omega_{\Gamma}$ as in (Moës et al., 1999). The resulting displacement field is displayed in Fig. 12. The crack opening is now clearly seen, since the functional basis includes discontinuous features across the crack. Let us note that the uncertainty level along the crack face is much higher than elsewhere because the initial determination of the displacement does not take into account the discontinuity.

\subsection{Normalization}

Even if the best measured displacement field is obtained, the bilinear or extended interpolation decreases the quality of the determination of stress intensity factors. The VCE field (be it optimal or not) is designed for extracting stress intensity factors from the asymptotic solution of a cracked body. Figure 13 illustrates this point. When stress intensity factors are computed by using a discretized VCE field, with an analytical auxiliary field and an analytical (i.e.Westergaard) displacement field such that $K_{I}$ is equal to $1 \mathrm{MPa} \sqrt{\mathrm{m}}$, one cannot distinguish the error on $K_{I}$. On the contrary, when the displacement field is such that exact nodal values are prescribed but a finite element

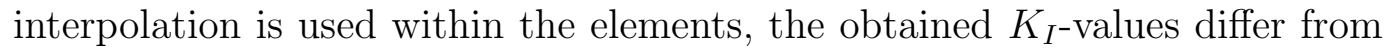
the exact solution $1 \mathrm{MPa} \sqrt{\mathrm{m}}$. For this reason, a normalization step of the VCE field is performed in order to obtain the exact value of stress intensity factors when they are computed from an exact displacement field interpolated on the discretization basis. 


\subsection{Identification results}

The values obtained for $K_{I}$ and $K_{I I}$ with this consistent displacement field and both conical and optimal weight functions are plotted in Fig. 14 as a function of the size of the integration domain. The stress intensity factors estimation using the interaction integral is theoretically domain independent. This is observed for the conical weight function as well as for the optimal one from a domain size of about $400 \mu \mathrm{m}$. In the range where the domain independence is observed, the values of the stress intensity factors for the conical and the optimal weight function are similar, namely, about $2.7 \pm 0.2 \mathrm{MPa} \sqrt{\mathrm{m}}$ and $2.6 \pm 0.1 \mathrm{MPa} \sqrt{\mathrm{m}}$ respectively for $K_{I}, 0.0 \pm 0.2 \mathrm{MPa} \sqrt{\mathrm{m}}$ and $0.0 \pm 0.2 \mathrm{MPa} \sqrt{\mathrm{m}}$ respectively for $K_{I I}$. These values are in agreement with those obtained by Roux and Hild (2006) (i.e. $K_{I}=2.3 \pm 0.15 \mathrm{MPa} \sqrt{\mathrm{m}}$ and $K_{I I}=0.05 \pm 0.05 \mathrm{MPa} \sqrt{\mathrm{m}}$ ) using a very different strategy (least squares fit to a combination of elastic fields). Furthermore, the latter strategy suffers from a slight dependency over the specific choice of fields a priori selected. This observation is a further motivation to follow the present approach, which is constructed to be domain independent and optimized in the sense of a reduction of the sensitivity to the measurement uncertainties.

The results obtained with the RSP function proposed by Rajaram et al. (2000) are compared to those of the optimized VCE in Fig. 15. The results of the RSP function are not as stable as those obtained with the optimized VCE. Variations as large as $0.8 \mathrm{MPa} \sqrt{\mathrm{m}}$ are observed. The mean values of the stress intensity factors are similar to those obtained with the optimized VCE but the domain independence of the stress intensity factors estimation with this function cannot be established. 


\subsection{Sensitivity to the crack tip location}

The noise robustness of the stress intensity factor identification is an important feature but the sensitivity to the crack tip location is also fundamental (Roux and Hild, 2006). As mentioned in the Introduction Section, the crack tip location is assumed to be known. However, this is often not the case, and the crack geometry has to be determined consistently with the evaluation of $K$. The interaction integral formalism has the potential of capturing the stress intensity factor and being less sensitive to the crack tip positioning than a least squares minimization because it relies on a domain-independent integral. Therefore, a sensitivity analysis is carried out.

In Fig. 16, the weight function optimisation reduces the relative error on $K_{I I}$ to $2 \%$ in the $\mathbf{x}$ direction and $4 \%$ in the $\mathbf{y}$ direction. In spite of an "accident" in the curve for a $\mathbf{y}$ mis-positioning, the relative error on $K_{I}$ is also significantly

decreased. This "accident" is explained by a topological reason. When the crack tip is moved from its initial position of 1 pixel in the $\mathbf{y}$ direction, a node of the cut elements initially above the crack is below afterwards. This explains the strong variation of $K_{I}$ for the optimal weight function that depends on the displacement functional basis and thus on the crack / mesh interactions.

\subsection{Vector weight function}

Up to now, the optimal weight function was assigned a direction parallel to the crack in the entire integration domain. Equation (2) only constrained the weight function direction on the crack faces and at the crack tip. This freedom has not been yet exploited. Therefore, an optimal vector weight function is 
obtained following the same procedure as for the scalar one. This vector weight function is plotted in Fig. 17 for mode $I$. Except in the vicinity of the crack, the optimal vector weight function does not remain parallel to the $\mathrm{x}$-direction, and both $x$ and $y$ components are of comparable order of magnitude.

Furthermore, Fig. 18 shows that the sensitivity to the crack tip position is reduced when compared to the scalar weight function and the relative error is now less than $5 \%$ in the $\mathbf{y}$ direction. The results concerning the noise sensitivity are also slightly improved. For a domain size of $700 \mu \mathrm{m}$, the ratio of the $K$ variances obtained for an optimal VCE vector as compared to the VCE cone reaches values as low as 0.012 for an uncorrelated noise to be compared to 0.017 for the optimal scalar VCE. The crack tip position sensitivity of the truncated cone weight function is also presented in Fig. 18. Although the truncated cone improved the noise robustness with respect to the conical one, the sensitivity to the crack tip position is much more important and makes this solution unreliable.

\section{Conclusion}

The paper proposes an improvement of the stress intensity factors identification by means of a digital image correlation technique and the interaction integral. This improvement concerns the noise robustness of this estimation. The kinematic field is always corrupted by a measurement uncertainty. Because of the space derivation, the displacement gradient displays higher uncertainty

levels and the computation of the interaction integral is then severely sensitive to noise.

The variance of the perturbation of the stress intensity factor for mode $I$ and 
mode $I I$ is obtained from a discrete derivation of the interaction integral. Since this variance is written as a quadratic form of the discrete VCE field, the minimization of the noise sensitivity is straightforward and leads to an optimization of the VCE field, tailored to the displacement functional basis, crack geometry and covariance of the noise. Then, depending on the measurement technique and on the noise characterization, an optimal VCE field is obtained for mode $I$ and mode $I I$.

The optimization of the weight function for an enhanced displacement functional basis (which seems to be a promising advance for digital image correlation techniques (Besnard et al., 2006; Réthoré et al., 2007)) provides a significant improvement to the noise robustness of the stress intensity factors determination, namely, the noise sensitivity is reduced up to a factor of about 100 for a domain size of $700 \mu \mathrm{m}$. Furthermore, the sensitivity to the presupposed crack tip positioning is also reduced by adopting a vector weight function. The relative variation of the stress intensity factors is down to $4 \%$ when the variation of the crack tip position keeps the crack / mesh interactions unchanged. It is also proven that those improvements (noise and crack tip location robustness) cannot be achieved by just truncating a cone weight function which is an easy but not optimal modification. The RSP function revealed to be close to the solution, yet less performing.

Beside the use of such a tool in the analysis of experimental data, which was the initial purpose, its implementation in finite element computations, is straightforward, and should exhibit essentially the same advantages as those reported above. Moreover, the optimal VCE being determined from the specific numerical discretization scheme, it allows one to benefit fully from the details of the mesh refinement without additional constraints on the mesh geometry. It 
may also easily be tailored to avoid spurious contributions from, e.g., plasticity occurring in the process zone, by imposing the VCE to be constant over the plastic domain, with an arbitrary shape of this domain. The numerical cost of the computation of the optimal VCE is typically marginal as compared to that of the primary problem solution.

\section{Acknowledgments}

This work was funded by the CETIM grant PROPAVANFIS: "Advanced methods for the experimental and numerical analysis of crack propagations under complex loadings". 


\section{References}

Anbanto-Bueno, J., Lambros, J., 2002. Investigation of crack growth in functionally graded materials using digital image correlation. Engineering Fracture Mechanics 69, 1695-1711.

Attigui, M., Petit, C., 1997. Mixed-mode separation in dynamic fracture mechanics: New path independent integrals. International Journal of Fracture 84 (1), 19-36.

Besnard, G., Hild, F., Roux, S., 2006. "Finite-element" displacement fields analysis from digital images: Application to Portevin-Le Châtelier bands. Experimental Mechanics 46, 789-803.

Forquin, P., Rota, L., Charles, Y., Hild, F., 2004. A method to determine the toughness scatter of brittle materials. International Journal of Fracture 125 (1), 171-187.

Goz, M., Dolbow, J., Moran, B., 1998. Domain integral formulation for stress intensity factor computation along curved three-dimensional interface cracks. International Journal of Solids and Structures 35 (15), 1763-1783.

Huntley, J.M., Field, J.E., 1989. Measurement of crack tip displacement field using laser speckle photography. Engineering Fracture Mechanics 30 (6), 779-790.

Marshall, D.B., Ratto, J.J., Lange, F.F., 1991. Enhanced Fracture Toughness in Layered Microcomposites of Ce- $\mathrm{ZrO}_{2}$ and $\mathrm{Al}_{2} \mathrm{O}_{3}$. Journal of the American Ceramic Society 74 (12), 2979-2987.

McNeill, S., Peters, W., Sutton, M., 1987. Estimation of stress intensity factor by digital image correlation. Engineering Fracture Mechanics 28 (1), 101112.

Moës, N., Dolbow, J., Belytschko, T., 1999. A finite element method for crack 
growth without remeshing. International Journal for Numerical Methods in Engineering 46 (1), 133-150.

Moës, N., Gravouil, A., Belytschko, T., 2002. Non-planar 3d crack growth by the extended finite element and level sets. Part I: Mechanical model. International Journal for Numerical Methods in Engineering 53 (11), 25492568 .

Nose, T., Fujii, T., 1988. Evaluation of Fracture Toughness for Ceramic Materials by a Single-Edge-Precracked-Beam Method. Journal of the American Ceramic Society 71 (5), 328-333.

Pancheri, P., Bosetti, P., Dal Maschio, R. Sglavo, V.M., 1998. Production of Sharp Cracks in Ceramic Materials by Three-Point Bending of Sandwiched Specimens. Engineering Fracture Mechanics 59 (4), 447-456.

Rajaram, H. and Socrate, S. and Parks, DM., 2000. Application of domain integral methods using tetrahedral elements to the determination of stress intensity factors. Engineering Fracture Mechanics 66 (5), 455-482.

Réthoré, J., Gravouil, A., Combescure, A., 2005a. An energy conserving scheme for dynamic crack growth with the extended finite element method. International Journal for Numerical Methods in Engineering 63, 631-659.

Réthoré, J., Gravouil, A., Morestin, F., Combescure, A., 2005b. Estimation of mixed-mode stress intensity factors using digital image correlation and an interaction integral. International Journal of Fracture 132 (1), 65-79.

Réthoré, J., Roux, S., Hild, F., 2007. From pictures to extended finite elements: Extended digital image correlation (X-DIC), C.R. Mecanique, accepted.

Roux, S., Hild, F., 2006. Stress intensity factor measurement from digital image correlation: post-processing and integrated approaches. International Journal of Fracture, 140 (1-4), 141-157.

Suo, X., Combescure, A., 1992. On the application of the $\mathcal{G} \theta$ method and its 
comparison with de Lorenzi's approach. Nuclear Engineering and Design $135,207-224$.

Yoneyama, S., Morimoto, Y., Takashi, M., 2006. Automatic evaluation of mixed-mode stress intensity factors utilizing digital image correlation. Strain $42,21-29$. 


\section{List of Figures}

1 Mesh (gray grid) and crack (solid black line) geometry used in the application. The domain used for the interaction integral is shown as a gray square centered on the crack tip.

$2 q$ function for the cone weight function $\mathbf{q}=q \mathbf{x}$. The crack tip is located at the right end of the gray line.

3 Enrichment strategy: circles denote $\mathcal{N}_{\text {cut }}$, squares $\mathcal{N}_{\text {tip }}$.

$4 q$ function for optimal weight function $\mathbf{q}=q \mathbf{x}$ for modes $I$ (a) and $I I(\mathrm{~b})$. The crack tip is located at the right end of the gray line.

5 Noise sensitivity reduction from the cone to the optimal VCE, for modes $I$ and $I I$.

6 Comparison of the optimal VCE for mode $I$, the RSP and conical VCEs in the plane $x=0$.

$7 \quad$ Noise sensitivity for mode $I$ with the conical, RSP and optimal weight functions.

8 Noise sensitivity reduction for different cone weight functions.

9 Noise sensitivity reduction for different noise correlations. 
10 Geometry of the sandwiched beam set-up (a). The brittle sample $(\mathrm{N})$ is put in between two steel beams (A and B). A three-point bend test is performed. A first stable crack is initiated. Initial (b) and cracked (c) $\mathrm{SiC}$ sample face. In the middle of the lower side, the black spot is the mark of the notch, from which a crack propagates. The image size is $1008 \times 1016$ pixels with a conversion factor $p=1.85 \mu \mathrm{m} /$ pixel.

11 Measured (a) $u_{x}$, and (b) $u_{y}$ displacement fields.

12 Projected (a) $u_{x}$ displacement, (b) $u_{y}$ displacement onto the extended functional basis.

13 Stress intensity factors computed from an exact displacement field are well captured (o and + ). The discretization error of the displacement field alters significantly the estimation with both conical $(\triangle)$ and optimal $(\times)$ VCE. This effect is corrected for through a renormalization of the VCE.

14 Stress intensity factor for the conical and optimal weight functions.

15 Stress intensity factor for the RSP and optimal weight functions.

16 Sensitivity for mode $I$ (a) and mode $I I$ (b) to the crack tip location.

17 Optimal vector weight function for mode $I$. The arrows give the orientation of the VCE and the contours its norm. 
18 Sensitivity to the crack tip location comparison with a vector weight function. 


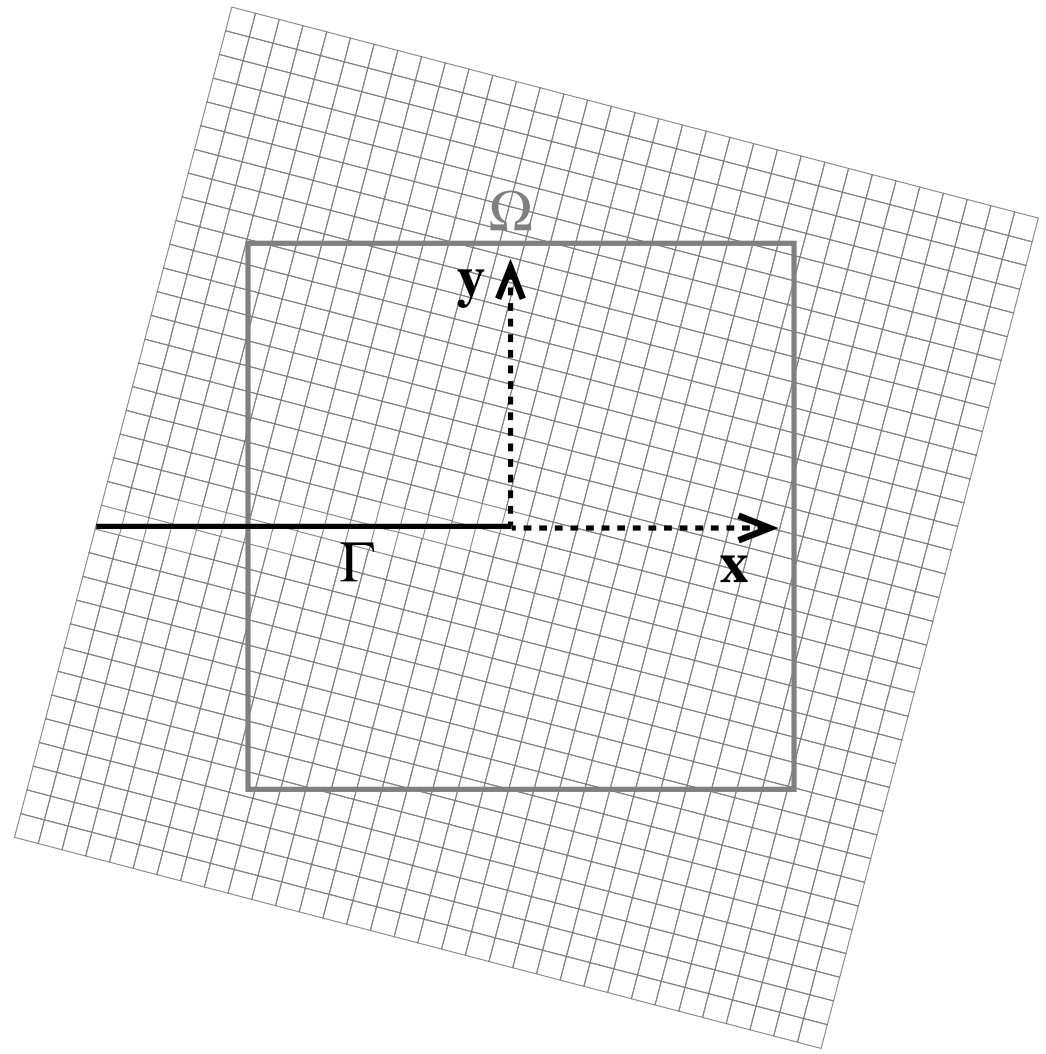

Fig. 1. Mesh (gray grid) and crack (solid black line) geometry used in the application. The domain used for the interaction integral is shown as a gray square centered on the crack tip. 


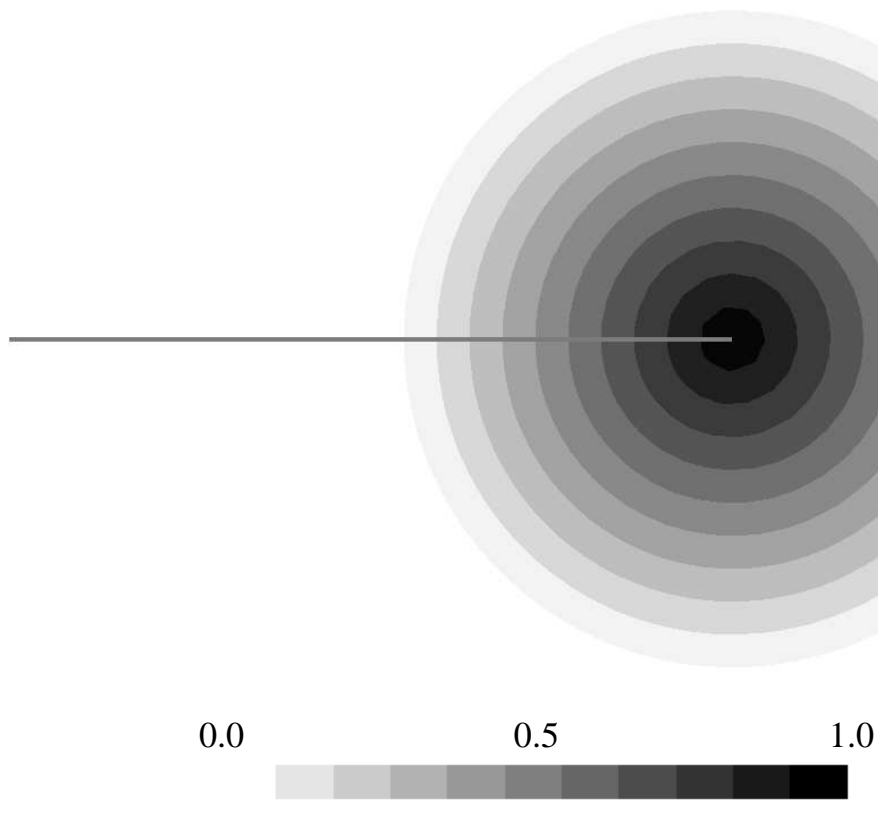

Fig. 2. $q$ function for the cone weight function $\mathbf{q}=q \mathbf{x}$. The crack tip is located at the right end of the gray line.

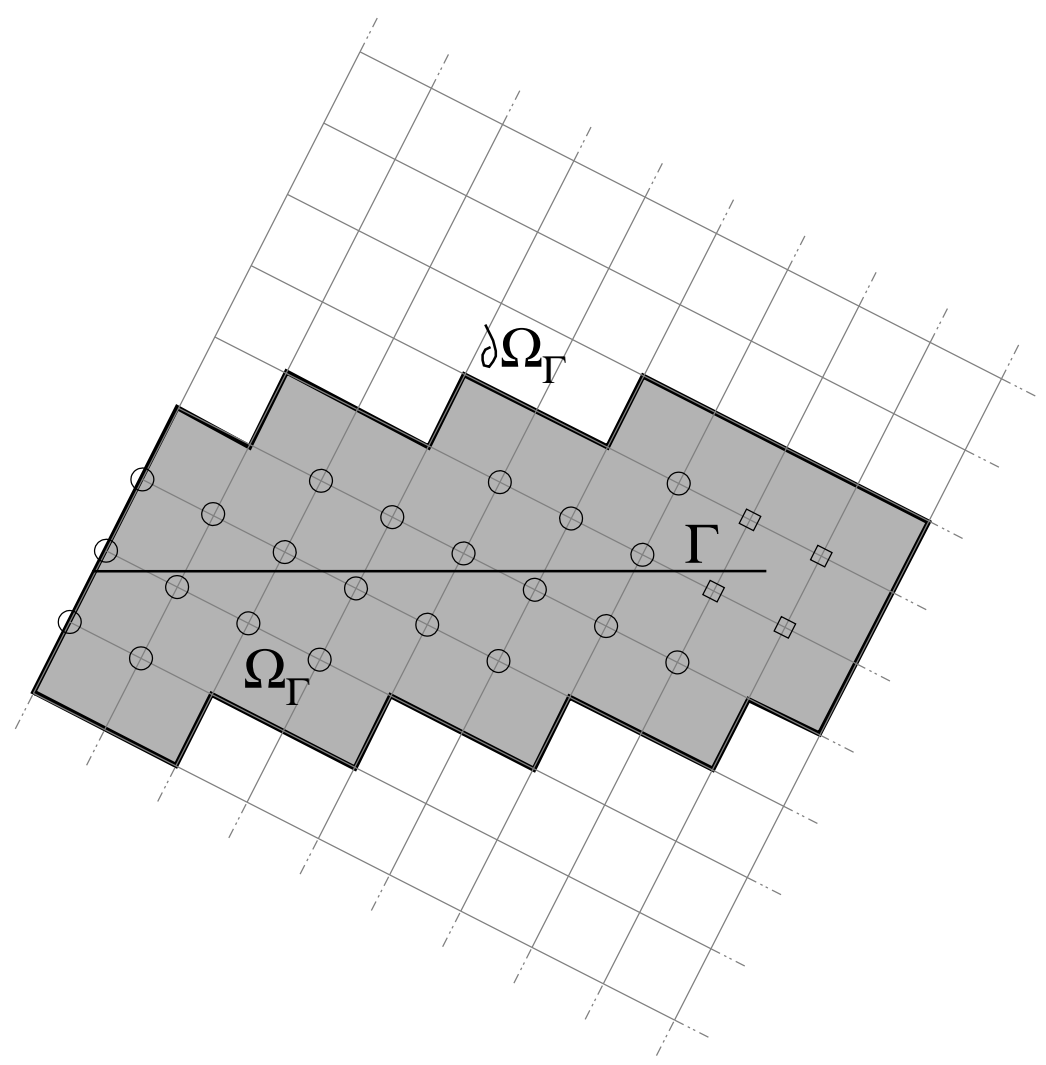

Fig. 3. Enrichment strategy: circles denote $\mathcal{N}_{\text {cut }}$, squares $\mathcal{N}_{\text {tip }}$. 

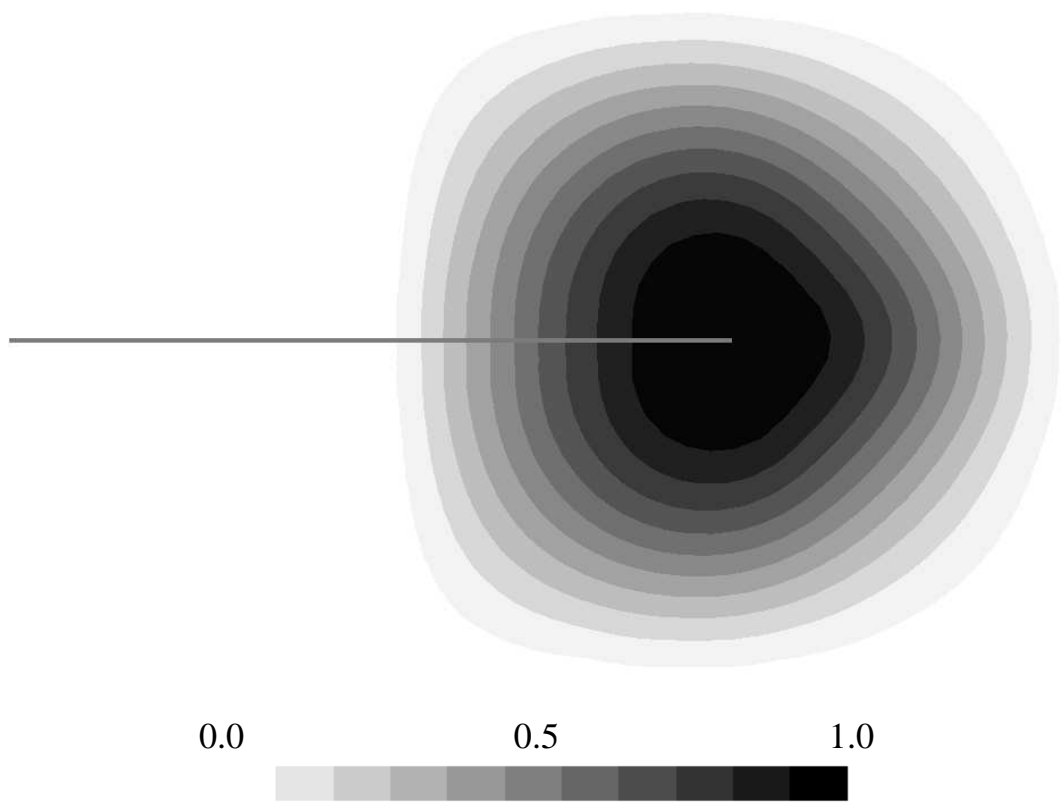

$-a-$

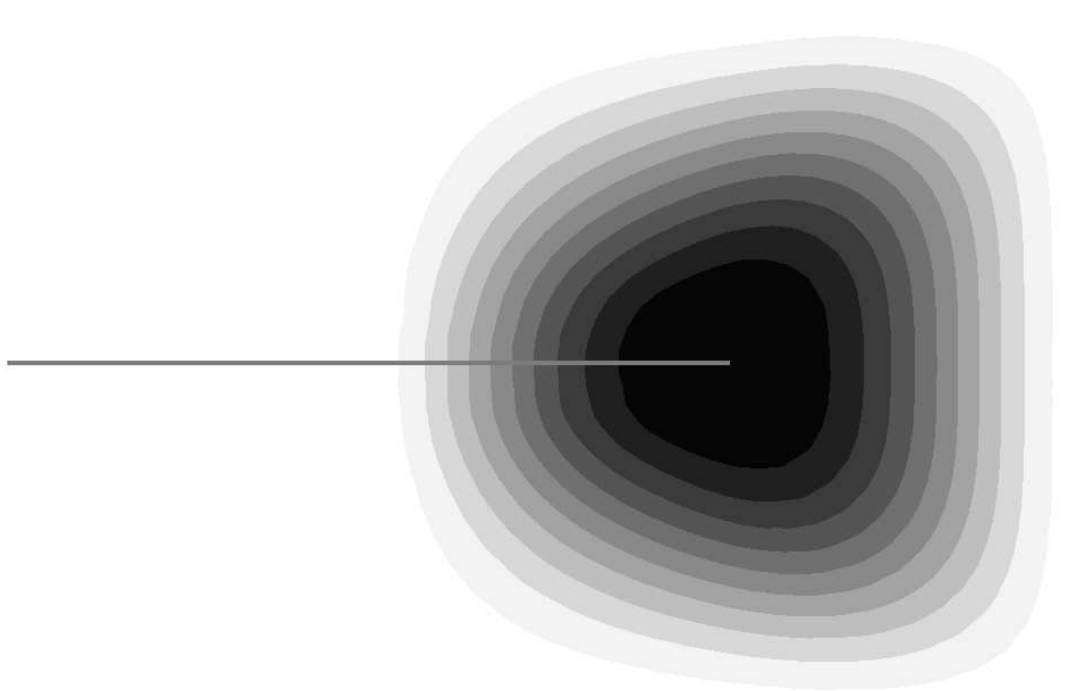

0.0

0.5

1.0

$-b-$

Fig. 4. $q$ function for optimal weight function $\mathbf{q}=q \mathbf{x}$ for modes $I$ (a) and $I I$ (b). The crack tip is located at the right end of the gray line. 


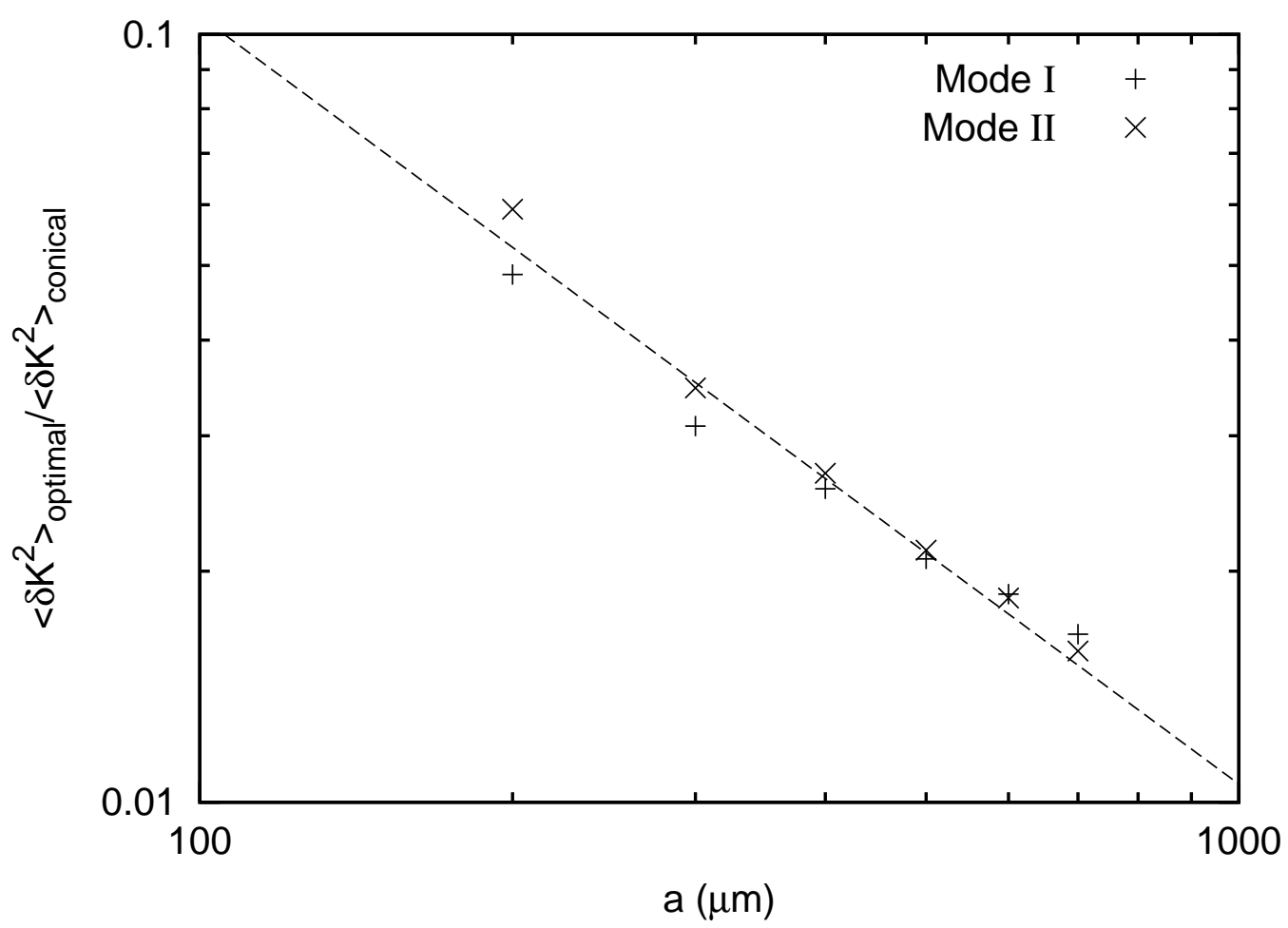

Fig. 5. Noise sensitivity reduction from the cone to the optimal VCE, for modes $I$ and $I I$.

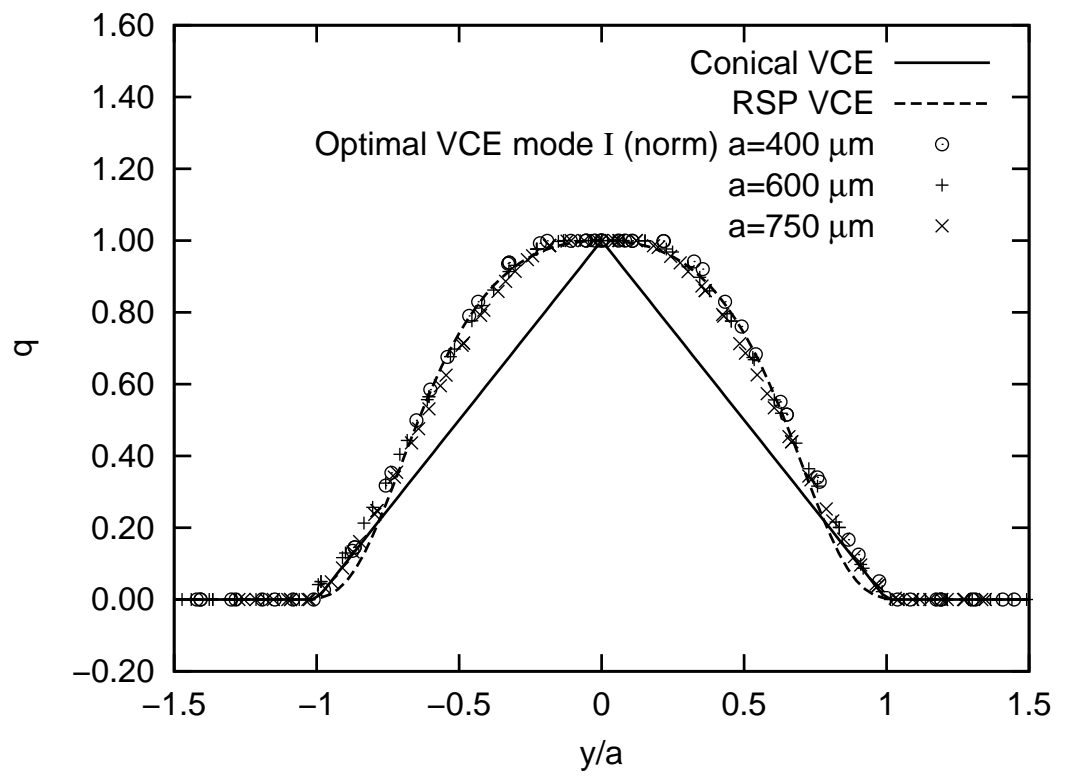

Fig. 6. Comparison of the optimal VCE for mode $I$, the RSP and conical VCEs in the plane $x=0$. 


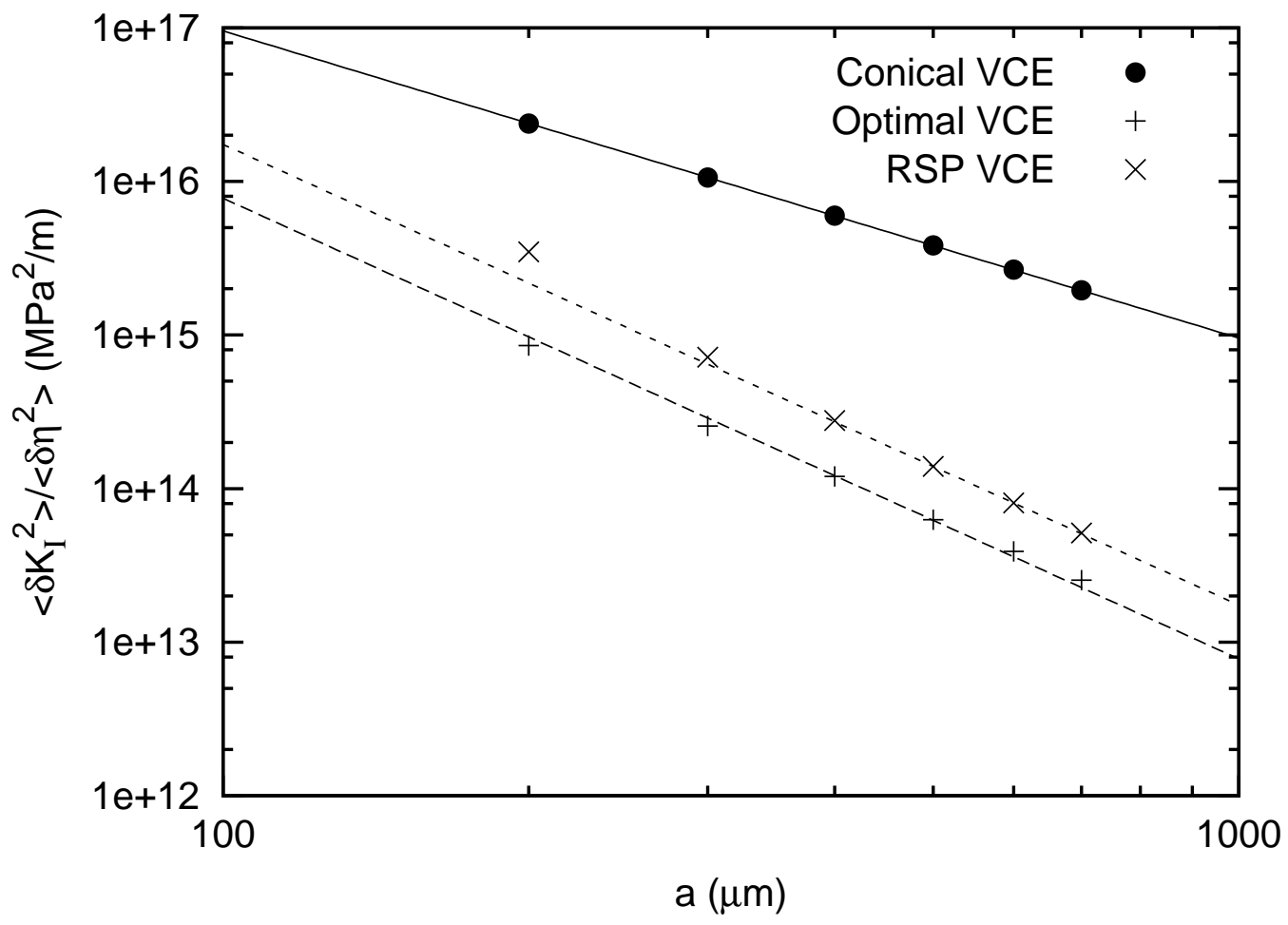

Fig. 7. Noise sensitivity for mode $I$ with the conical, RSP and optimal weight functions. 


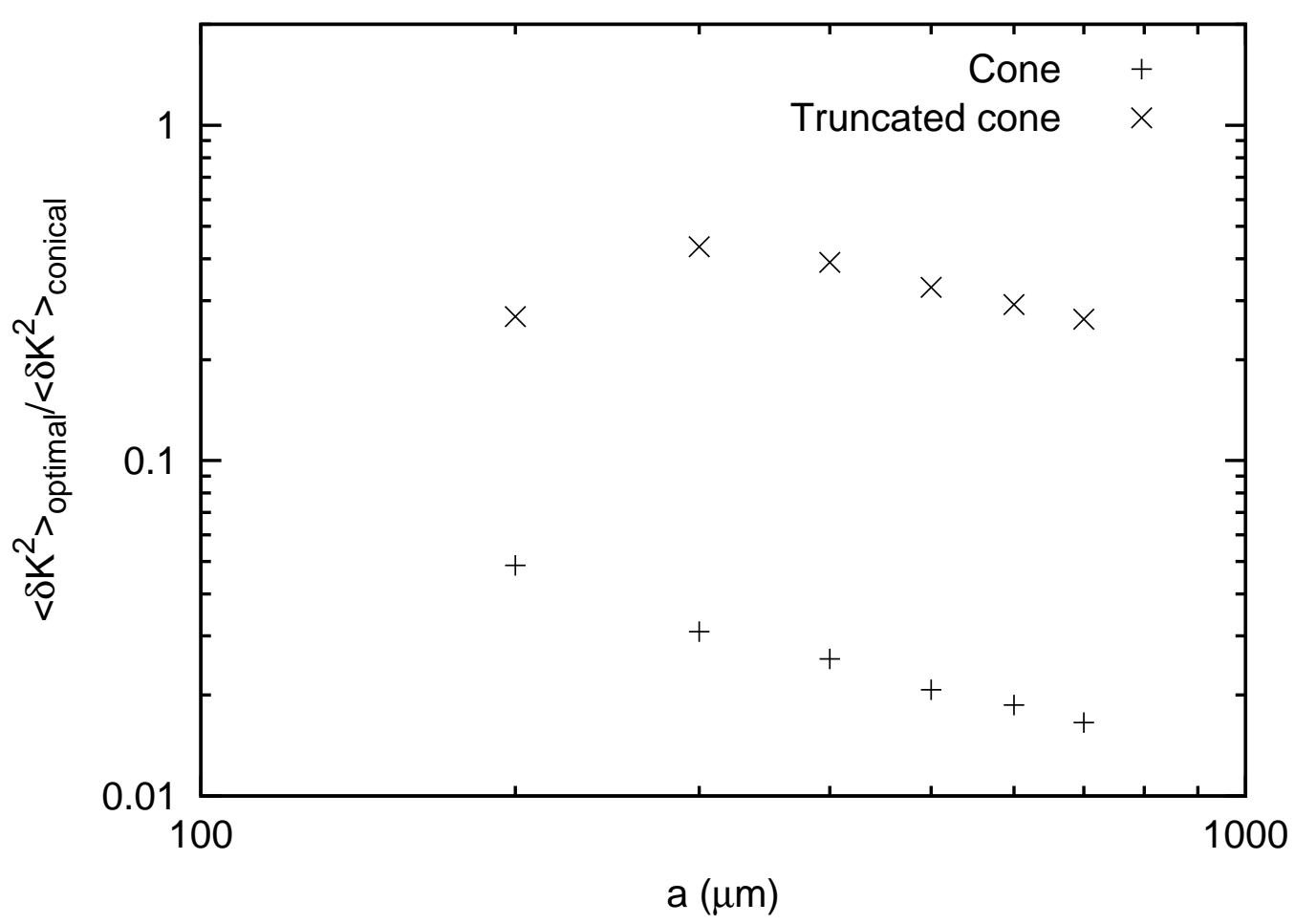

Fig. 8. Noise sensitivity reduction for different cone weight functions.

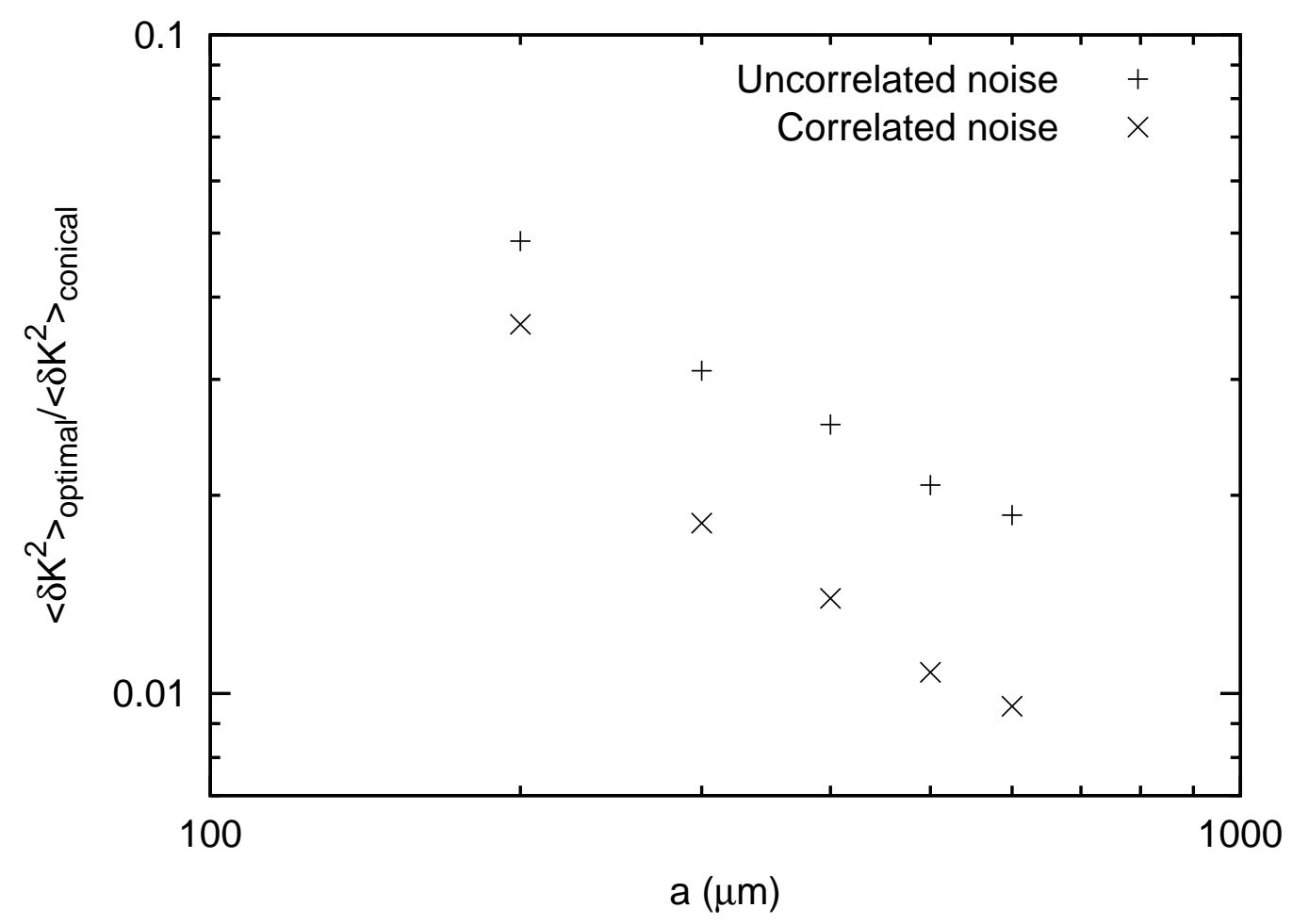

Fig. 9. Noise sensitivity reduction for different noise correlations. 


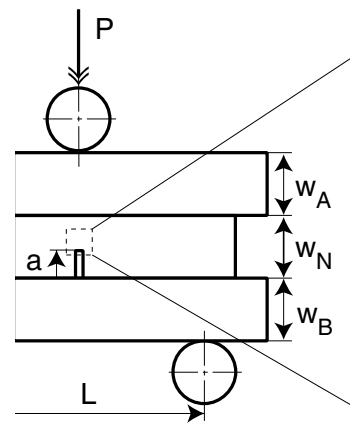

$-a-$

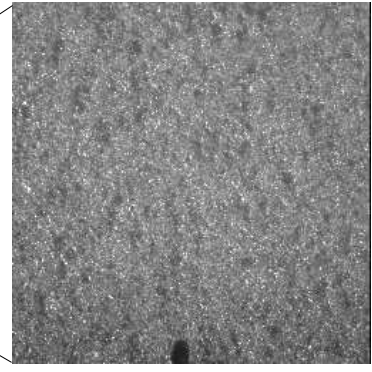

$-b-$

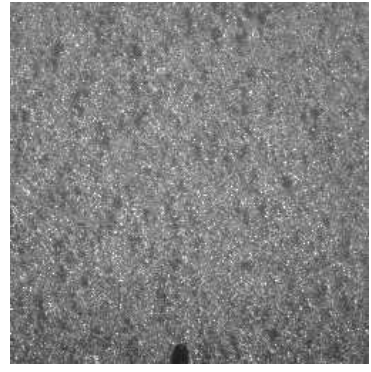

$-\mathrm{C}-$

Fig. 10. Geometry of the sandwiched beam set-up (a). The brittle sample (N) is put in between two steel beams (A and B). A three-point bend test is performed. A first stable crack is initiated. Initial (b) and cracked (c) SiC sample face. In the middle of the lower side, the black spot is the mark of the notch, from which a crack propagates. The image size is $1008 \times 1016$ pixels with a conversion factor $p=1.85 \mu \mathrm{m} /$ pixel.
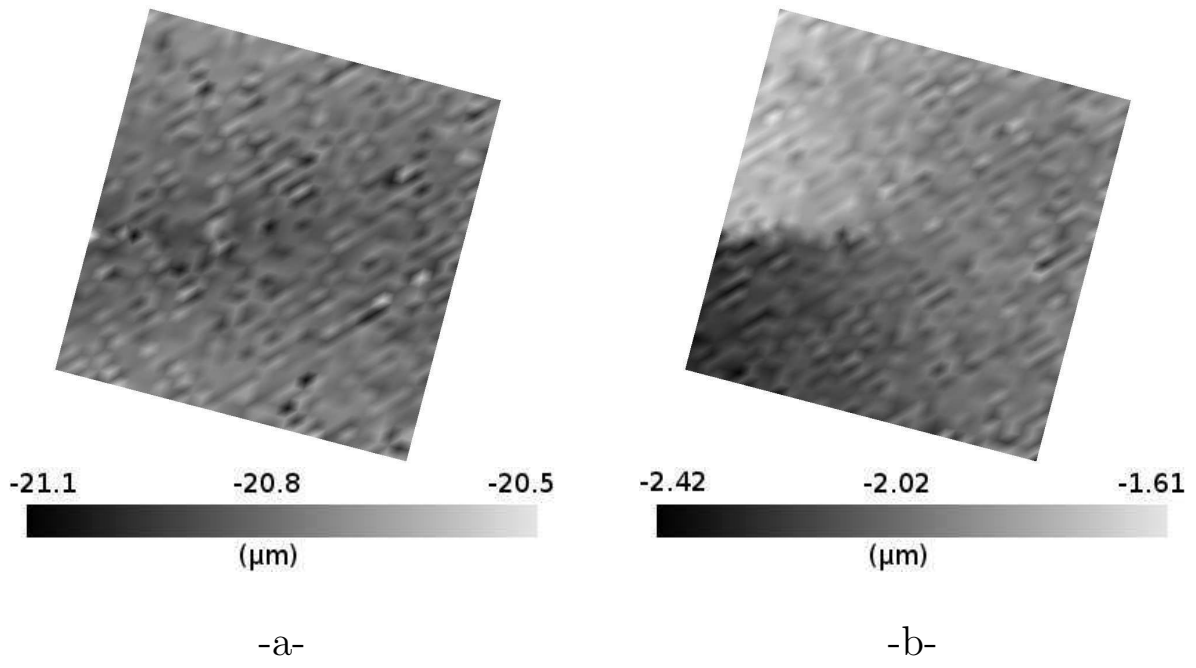

Fig. 11. Measured (a) $u_{x}$, and (b) $u_{y}$ displacement fields. 

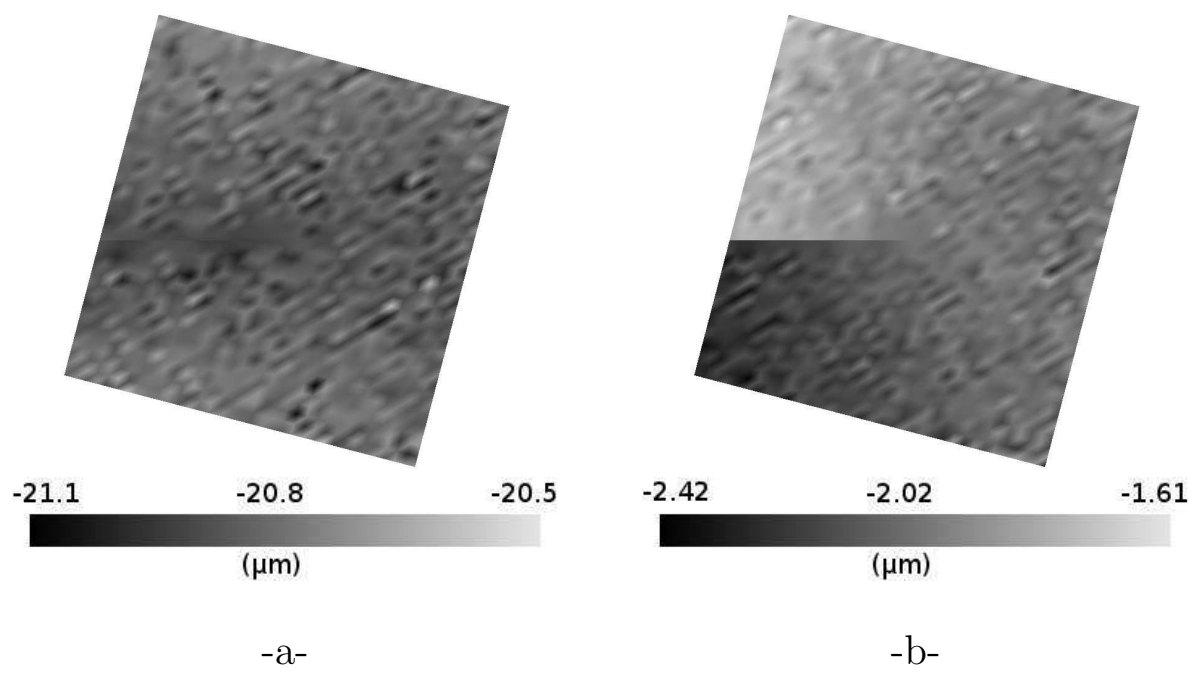

Fig. 12. Projected (a) $u_{x}$ displacement, (b) $u_{y}$ displacement onto the extended functional basis.

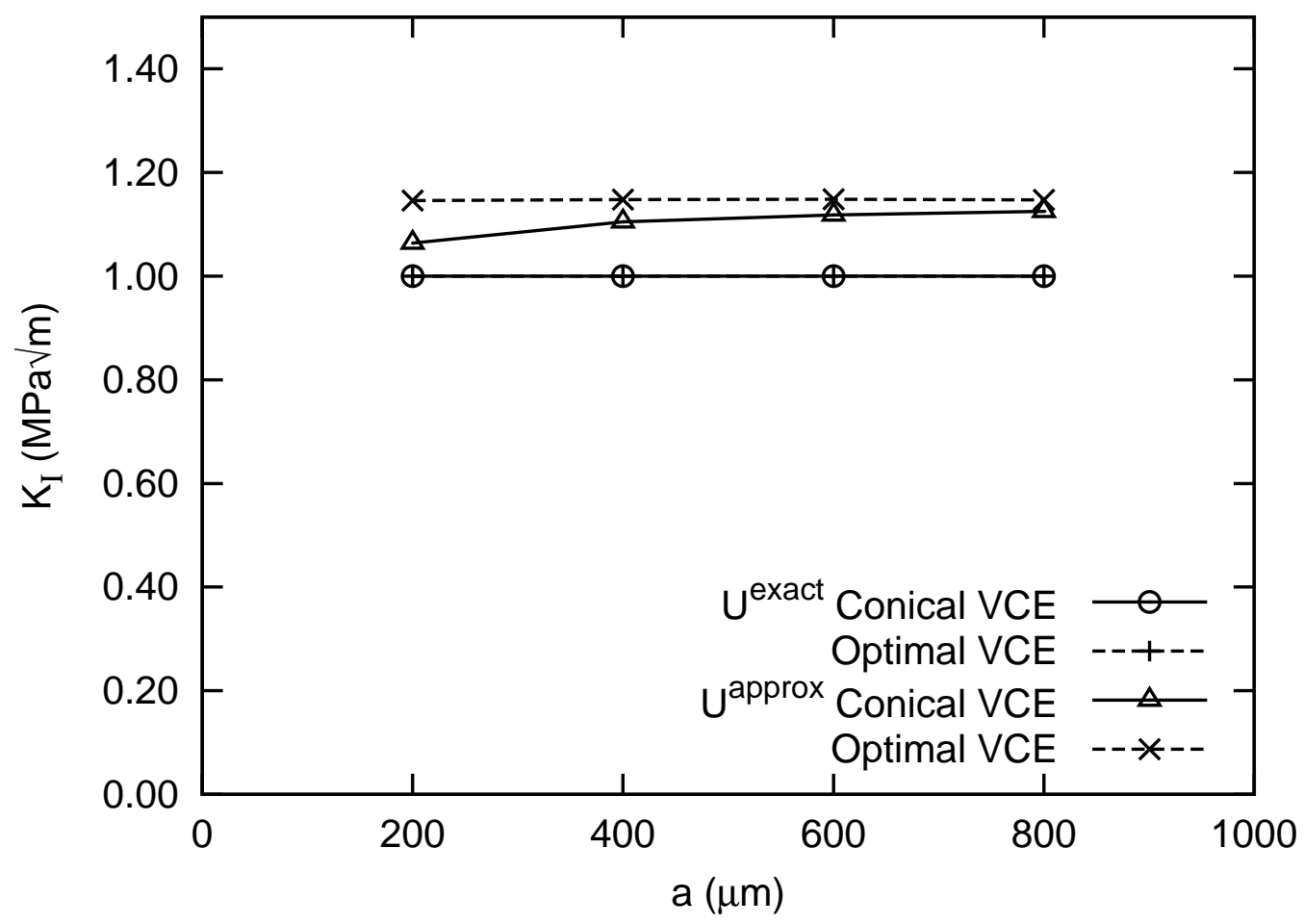

Fig. 13. Stress intensity factors computed from an exact displacement field are well captured $(\circ$ and + ). The discretization error of the displacement field alters significantly the estimation with both conical $(\triangle)$ and optimal $(\times)$ VCE. This effect is corrected for through a renormalization of the VCE. 


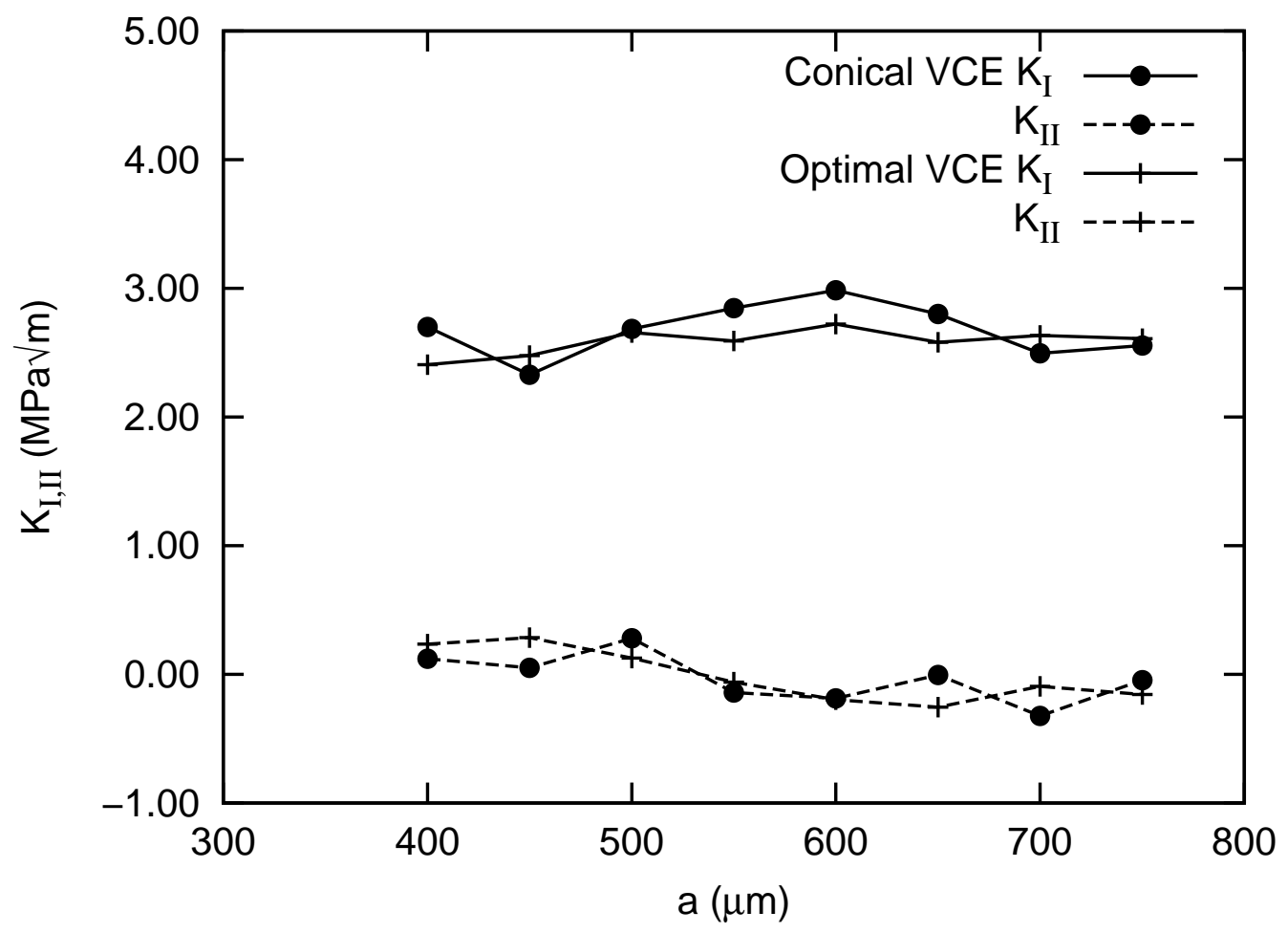

Fig. 14. Stress intensity factor for the conical and optimal weight functions.

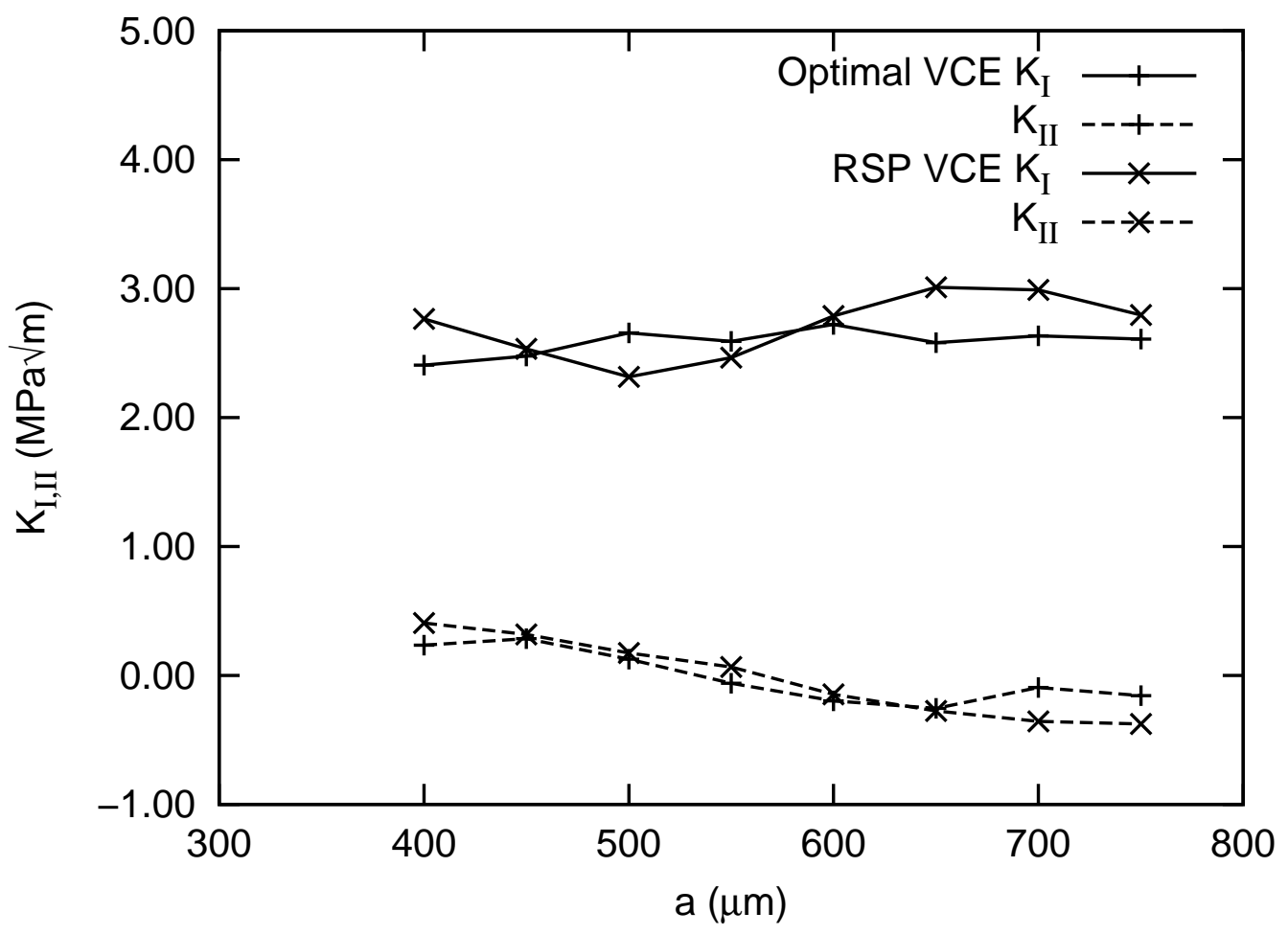

Fig. 15. Stress intensity factor for the RSP and optimal weight functions. 

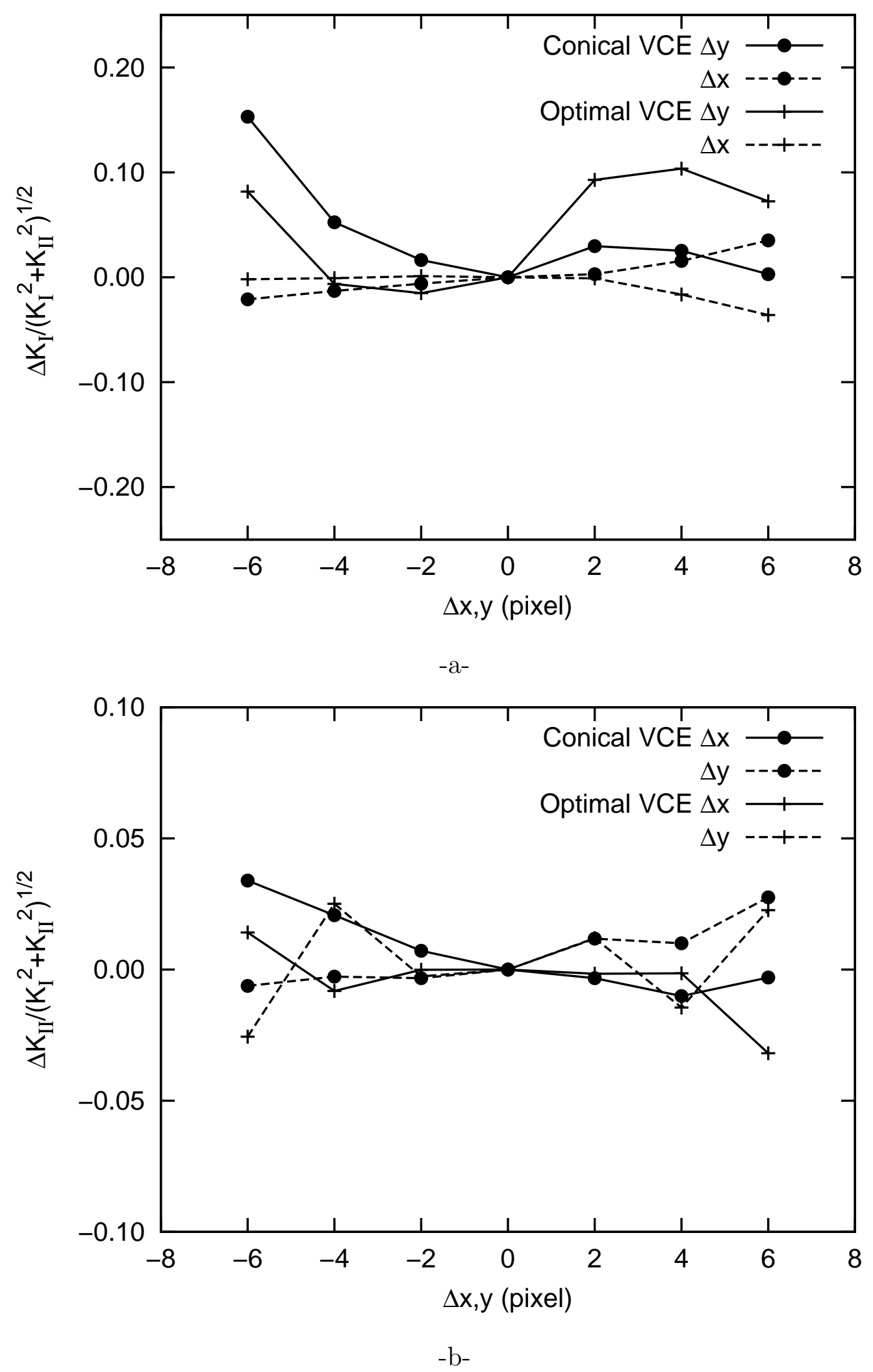

Fig. 16. Sensitivity for mode $I$ (a) and mode $I I$ (b) to the crack tip location. 

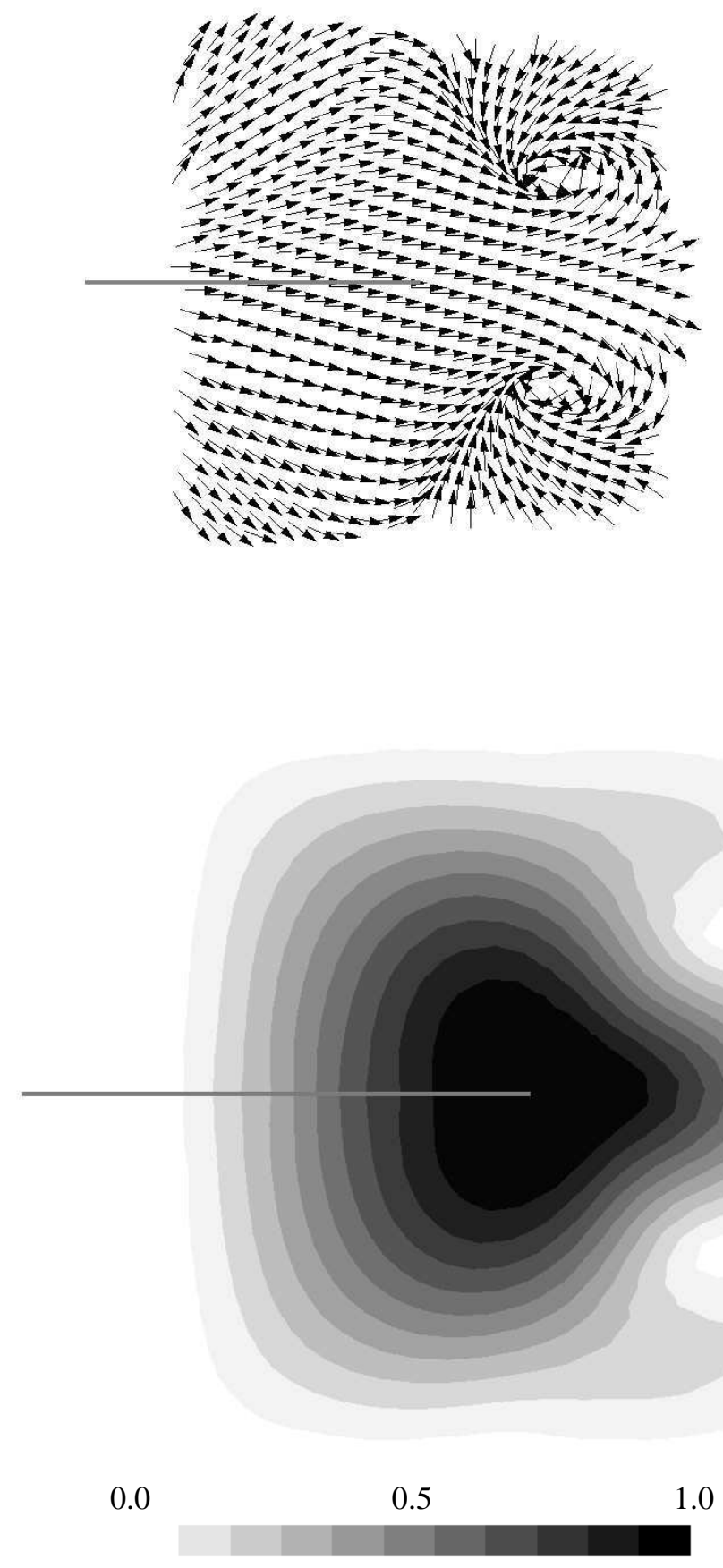

Fig. 17. Optimal vector weight function for mode $I$. The arrows give the orientation of the VCE and the contours its norm. 


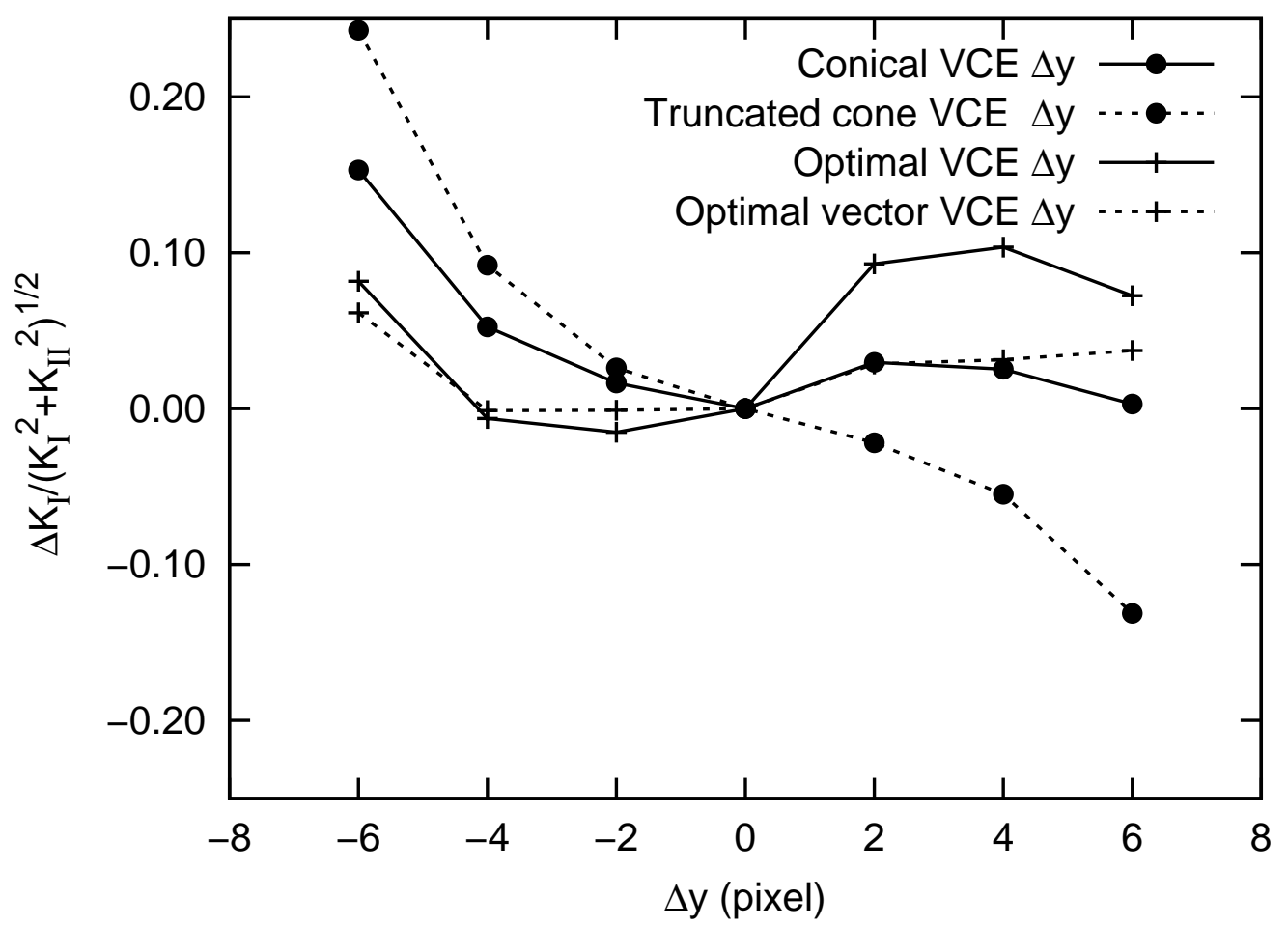

Fig. 18. Sensitivity to the crack tip location comparison with a vector weight function. 